$74 \times \sqrt{3}$

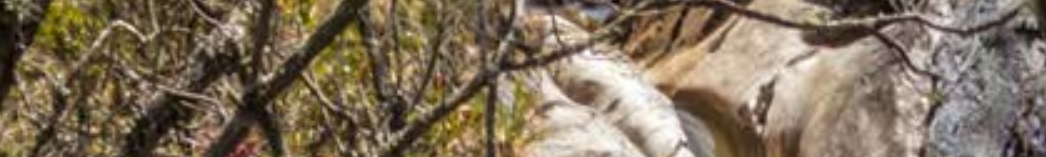
W.

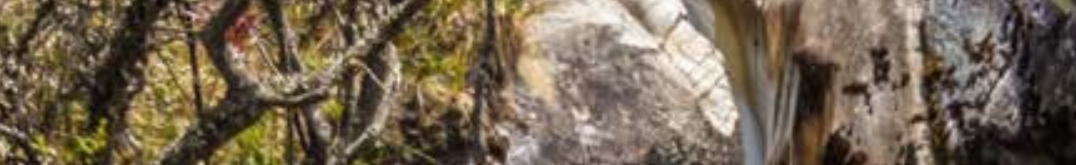

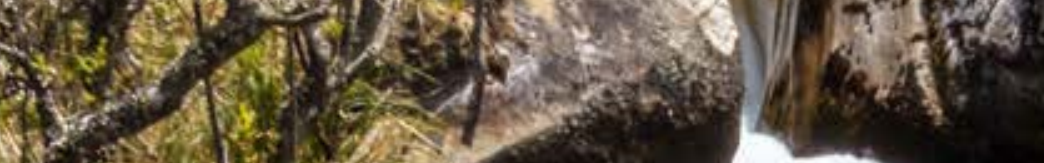
2. S.

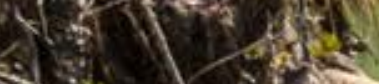 th

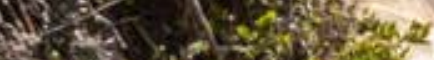 Tit}

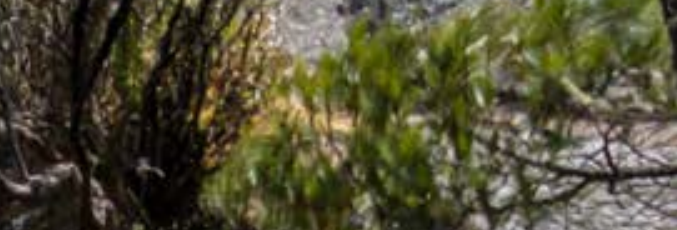

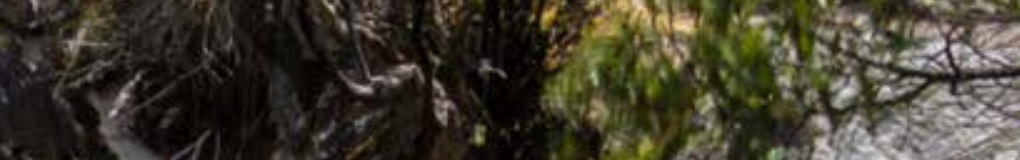

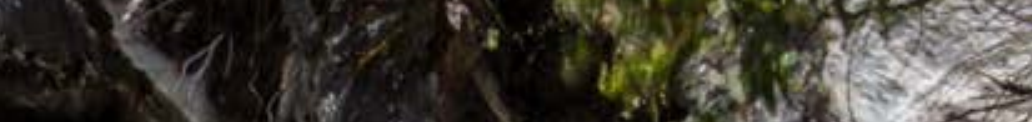
(n) a)<smiles>C#C[AsH]</smiles>
. $\sum_{3}$

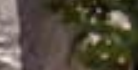

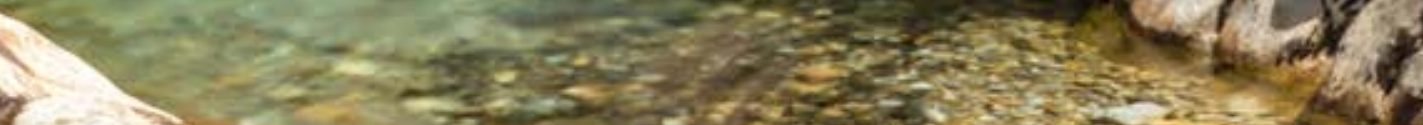




\title{
APORTE DE UN PROCEDIMIENTO DIDÁCTICO PARA MEJORAREL CONOCIMIENTO DE LA BIODIVERSIDAD EN SECUNDARIA
}

\section{Contribution of a Didactic Procedure to Improve Knowledge about Biodiversity in Junior High School}

\section{Contribuição de um procedimento didático para melhorar o conhecimento da biodiversidade na escola secundária inferior}

\author{
Omar García-Vázquez* \\ Norberto Sánchez-Veloz ** \\ Rodney Reinier García-Pérez ${ }^{\star \star \star}$
}

Fecha de recepción: 9 de abril del 2020

Fecha de aceptación: 20 de junio del 2020

\section{Cómo citar este artículo:}

García-Vázquez, O., Sánchez-Veloz, M. y García-Pérez, R. (2020). Aporte de un procedimiento didáctico para mejorar el conocimiento de la biodiversidad en secundaria básica. Bio-grafía: 13(25 ), 49-59. https://doi.org/10.17227/bio-grafia.vol.13.num25-11575

\section{Resumen}

El presente artículo de investigación muestra los resultados de la aplicación de un procedimiento didáctico para mejorar el conocimiento de la biodiversidad en los estudiantes de séptimo y octavo grado de la escuela secundaria básica urbana Juan Vitalio Acuña Núñez del municipio de Pilón, en la provincia de Granma, Cuba. Se tomó como escenario el área protegida Punta de Hicacos. Se siguió un enfoque descriptivo-explicativo y se trabajó desde el paradigma sociocrítico que considera la unidad dialéctica de lo teórico y lo práctico, como un todo inseparable. El procedimiento se estructuró en cinco fases: observación, explicación, interpretación, comprensión y control de la apropiación del aprendizaje. Entre las conclusiones se destaca que el procedimiento corrobora su pertinencia y efectividad para transformar los conocimientos, los sentimientos y las actitudes con relación a la biodiversidad del entorno natural donde los estudiantes viven y se desarrollan.

Palabras clave: educación ambiental; procedimiento didáctico; biodiversidad; educación para la conservación

\footnotetext{
* Doctor en Ciencias Pedagógicas y licenciado en Educación, especialidad Biología. Profesor titular, Universidad de Granma (Cuba). Correo electrónico: ogarciav@udg.co.cu. Orcid: https://orcid.org/0000-0002-0712-9734

* Doctor en Ciencias Pedagógicas y licenciado en Educación, especialidad Biología. Profesor titular, Universidad de Granma (Cuba). Correo electrónico: nsanchez@udg.co.cu

*** Licenciado en Educación, especialidad Biología. Metodólogo de Biología. Correo electrónico: reiniergarcia@pi.gr.rimed.cu
} 


\section{Abstract}

This research article shows the results of the application of a didactic procedure to improve the knowledge of biodiversity in seventh and eighth grade students of the Juan Vitalio Acuña Núñez urban basic secondary school in the municipality of Pilón, in the province of Granma, Cuba. The setting was the Punta de Hicacos protected area. The researchers followed a descriptive-explanatory approach and based upon the socio-critical paradigm which considers the dialectical unity of theory and practice, as an inseparable whole. The procedure was structured into five stages: observation, explanation, analysis, comprehension, and control of the appropriation of knowledge. Among the conclusions, it is highlighted that the procedure corroborates its relevance and effectiveness to transform knowledge, feelings and attitudes, in relation to the biodiversity of the natural environment where students live and develop.

Keywords: environmental education; didactic procedure; biodiversity; conservation education

\section{Resumo}

Este artigo de pesquisa mostra os resultados da aplicação de um procedimento didático para melhorar o conhecimento da biodiversidade em alunos da sétima e oitava séries da escola secundária básica urbana Juan Vitalio Acuña Núñez no município de Pilón, na província de Granma, Cuba. O cenário era a área protegida de Punta de Hicacos. Foi seguida uma abordagem descritivaexplicativa, trabalhando a partir do paradigma sócio-crítico que considera a unidade dialética do teórico e do prático como um todo inseparável. O procedimento foi estruturado em cinco fases: observação, explicação, interpretação, compreensão e controle da apropriação da aprendizagem. Entre as conclusões, destaca-se que o procedimento corrobora sua relevância e eficácia na transformação do conhecimento, sentimentos e atitudes em relação à biodiversidade do ambiente natural onde os estudantes vivem e se desenvolvem.

Palavras-chave: educação ambiental; procedimento didático; biodiversidade; educação para a conservação. 
El reto de explorar las potencialidades de la biodiversidad y su relación con la enseñanza de la biología en un espacio en interacción directa con la variabilidad de lo vivo constituye un insumo para la construcción del conocimiento y una mirada posible desde la pedagogía y el saber pedagógico (Herrera, 2020). De ahí la necesidad de lograr que la biología deje de ser únicamente para aprender sobre la vida, y llegue a ser una ciencia que enseñe a vivir y actuar para la sostenibilidad de la vida. De esta manera, el profesor de biología sería un mediador entre, al menos, tres ámbitos: el científico, el cotidiano y el escolar (Castro y Valbuena, 2018).

Ante esta exigencia social, la escuela debe propender por garantizar la formación del sentido de pertenencia y responsabilidad del ser humano hacia y con la naturaleza y elevar la calidad del proceso de enseñanza-aprendizaje de la biología en la escuela media, para transformar su enseñanza, en función de lograr que el alumnado interiorice con mayor profundidad el sentido de del contenido de biodiversidad. Así, la educación se convierte en una herramienta imprescindible para adquirir valores tan necesarios como el respeto hacia los seres vivos y el medio ambiente (Santos-Ellakuria, 2019).

De ahí que se han de proponer herramientas didácticas que favorezcan la superación de los docentes, para que faciliten cambios conceptuales respecto al modelo de enseñanza que practica el profesor de biología en la Escuela Secundaria Básica, cambios metodológicos en su saber hacer práctico y, en particular, lograr transformaciones en la manera de tratar el contenido de biodiversidad para perfeccionar su enseñanza y aprendizaje. De modo que "si queremos, armonizar la relación entre las personas y la biodiversidad, será necesario prestar cada vez más atención a la dimensión humana de la conservación ambiental, y así evitar convertir el patrimonio biológico en un tesoro perdido" (Álvarez, 2001, p. 9).

Por tanto, educar para la conservación exige desarrollar en el individuo conocimientos, procedimientos y valores, de modo que garanticen una actuación consecuente con respecto a las diferentes formas de vida que habitan en el planeta. Esta es una razón suficiente para apoyar que "la conservación de la biodiversidad se logrará en la medida en que ésta sea conocida, valorada adecuadamente y empleada de forma racional" (Programa de las Naciones Unidas para el Medio Ambiente, 2005, p. 10).

A pesar de lo anterior, es preciso señalar que, aunque en los programas de Biología en la escuela secundaria básica se tratan los contenidos relativos a la diversidad biológica y su problemática en torno a la educación para la conservación, en la práctica aún existen evidencias de insuficiencias. Es el caso, por ejemplo, de los métodos y procedimientos metodológicos empleados por los docentes en secundaria básica, que aún son insuficientes para generar en el educando un aprendizaje contextualizado de la biodiversidad local. Así pues, se estudia la representatividad de la biodiversidad cubana, pero se limita el tratamiento de sus dimensiones motivacional, afectiva, cultural, estética y su vulnerabilidad.

Persiste una limitada planificación de excursiones a la naturaleza, lo que incide en la vinculación de los estudiantes con la realidad ambiental, para potenciar el conocimiento y el respeto por la biodiversidad local, por lo cual queda incompleto su aprendizaje. Por otro lado, se constató la poca comprensión de los estudiantes sobre los bienes y servicios que ofrece la diversidad biológica. Además de lo dicho hasta ahora, hay que añadir que, en la práctica se observan comportamientos inadecuados en los estudiantes, que afectan la dinámica de los ecosistemas donde habitan las diversas formas de vida de la escuela y la comunidad donde viven y se desarrollan.

Así, llegados a este punto, vale la pena señalar que lo expuesto podría ser una muestra de algunas debilidades atribuibles al tratamiento del contenido de biodiversidad en secundaria básica. Este obedece, entre otras causas, a la limitada utilización por parte del docente de procedimientos con un enfoque investigativo que permitan una interconexión con el entorno, y a la poca planificación de excursiones a la naturaleza que motiven y acerquen al estudiante de manera directa a la realidad ambiental que lo rodea como escenario potencial de aprendizaje.

Ahora bien, con lo que hemos expresado como parte del diagnóstico inicial no queremos plantear que exista una incompetencia por parte de los profesores de biología de secundaria básica en el país. Muy por el contrario, estamos defendiendo la necesidad de perfeccionar la didáctica de la biodiversidad en este nivel para obtener mejores resultados en el aprendizaje de los estudiantes, a partir de que el docente utilice la realidad ambiental próxima que lo rodea para lograr una mayor motivación y acercamiento a la biodiversidad local.

De esta manera, el objetivo de la presente investigación es describir la construcción de un procedimiento didáctico de interconexión con el entorno ambiental, desarrollado a través de excursiones a la naturaleza en el área protegida de Punta de Hicacos, perteneciente al parque nacional Desembarco del Granma, Patrimonio de la Humanidad en la costa sur de Cuba, para la educación para la conservación de la biodiversidad. 


\section{Materiales y métodos}

Para llevar a cabo la investigación, se emplearon métodos propios del nivel teórico del conocimiento, como el analítico-sintético, inductivo-deductivo y el enfoque de sistema. Estos métodos proporcionaron los elementos necesarios para el análisis del objeto de la investigación y la sistematización de la información sobre el tema, así como para la determinación del marco teórico referencial, la interpretación y el análisis de la información obtenida. Algunos de los métodos empíricos utilizados fueron: análisis documental, entrevista, encuestas y observación; estos permitieron conocer el estado inicial del problema.

La investigación es de carácter descriptivo-explicativo y se trabaja desde el paradigma sociocrítico que considera la unidad dialéctica de lo teórico y lo práctico, como un todo inseparable. Pretende comprender de manera más consistente la teoría y la práctica educativa, considerando al estudiante como investigador. Desde este paradigma, los problemas de investigación parten de situaciones reales y tienen por objeto de estudio transformar la práctica; su selección la realiza el propio grupo, que cuestiona la situación inicial (según complementan Gil Álvarez et al., 2017).

Este paradigma se enmarca en la investigación-acción participativa (IAP), que constituye una opción metodológica de mucha riqueza, ya que, por una parte, permite la expansión del conocimiento, y, por la otra, produce respuestas concretas a problemáticas que se plantean los investigadores y coinvestigadores cuando deciden abordar una interrogante, temática de interés o situación problemática y desean aportar alguna alternativa de cambio o transformación (Colmenares, 2011).

Se contó con una muestra de 34 estudiantes de séptimo y octavo grado de la secundaria básica urbana Juan Vitalio Acuña Núñez del municipio de Pilón, en la provincia de Granma, con aptitudes distintas. Se destacan en ellos talentos para el dibujo, la expresión corporal, la narrativa, la oralidad y una actitud en general curiosa, proactiva y propositiva. La muestra la componen 16 mujeres y 18 hombres, con edades comprendidas entre los 12 y 15 años de edad.

La experiencia se desarrolla en la costa sur de Cuba, específicamente en el área protegida Punta de Hicacos, perteneciente al parque nacional Desembarco del Granma, donde predomina una vegetación de costa arenosa y amplia biodiversidad. Se seleccionó este escenario por encontrarse cerca de la escuela y los barrios aledaños donde viven los estudiantes de la muestra, así como por la riqueza de paisajes naturales muy variados, donde se puede disfrutar también de la presencia de muchas espe- cies de la fauna cubana, aunque no todas son fácilmente visibles, como el manatí, el tocororo, la pedorrera o cartacuba y el carpintero churroso, entre otras.

Se establecen tres dimensiones: cognitiva, procedimental y conductual, con sus respectivos indicadores. Estas fueron conformadas después de contrastar aspectos conceptuales y características de la actividad (cognoscitiva, práctica, valorativa y comunicativa) del estudiante respecto a la biodiversidad para evaluar las transformaciones alcanzadas.

\section{Dimensión cognitiva}

Se tuvieron en cuenta los siguientes indicadores:

- Conocimiento de los problemas ambientales a nivel global, provincial y local.

- Conocimiento del concepto de biodiversidad y conservación.

- Conocimiento de la cultura popular comunitaria y de los saberes tradicionales.

- Conocimiento de los bienes y servicios que ofrece la biodiversidad.

- Conocimiento de las causas de la pérdida de la biodiversidad y sus consecuencias.

- Conocimiento de las medidas para minimizar las afectaciones.

\section{Dimensión procedimental}

Se tuvieron en cuenta los siguientes indicadores:

- Desarrollo de las habilidades de investigación práctica.

- Habilidades para la interpretación crítica y la toma de decisiones ante los problemas ambientales.

- Habilidades para la búsqueda y el procesamiento de la información con el empleo de las nuevas tecnologías de la información y las comunicaciones.

\section{Dimensión conductual}

Se tuvieron en cuenta los siguientes indicadores:

- Relaciones armónicas con las diferentes formas de vida.

- Responsabilidad, asunción de actitudes y toma de decisiones, espíritu crítico y autocrítico ante comportamientos inadecuados con las diferentes formas de vida en el entorno escolar y comunitario.

- Manifestaciones de sentimientos de amor por la biodiversidad cubana, respeto, motivación, sensibilidad e implicación como actores proactivos. 


\section{Métodos de enseñanza en la biología y procedimientos}

Así las cosas, para explicar los contenidos biológicos en secundaria básica, métodos como la observación y la experimentación deben estar presentes en cualquier clasificación que se adopte. Sin embargo, en un proceso de enseñanza-aprendizaje orientado a ofrecer tratamiento a contenidos de biodiversidad, son los métodos problémicos los que tienen su espacio por excelencia, ya que su esencia se corresponde con las contradicciones propias del contenido que se analiza.

Dentro de los métodos problémicos están: la exposición problémica, la búsqueda parcial, la conversación heurística y el método investigativo, que posibilitan la función protagónica del estudiante en el proceso de enseñanza-aprendizaje. La esencia del método investigativo radica en que los alumnos, guiados por el docente, se introducen en el proceso de búsqueda de solución a problemas nuevos para ellos, a fin de adquirir la nueva experiencia de la actividad creadora. En otras palabras, un mismo método puede integrar variados procedimientos en correspondencia con las características en que este se desarrolla.

De acuerdo con Silvestre (2003), los procedimientos son

complemento de los métodos de enseñanza, constituyen herramientas que le permiten al docente orientar y dirigir la actividad del escolar en colectividad de modo que la influencia de otros propicie el desarrollo individual estimulando el pensamiento lógico, el pensamiento teórico y la independencia cognoscitiva motivándolos a pensar en un clima favorable de aprendizaje. (p. 26)

Labarrere y Valdivia agregan que estos "constituyen un detalle del método, es decir, es una operación particular, práctica o intelectual de la actividad del profesor o de los estudiantes, la cual complementa la forma como los estudiantes asimilan los conocimientos que presupone determinado método" (1989, p. 48).

En ese orden de ideas, y de acuerdo con Novo (1998), desde lo ambiental los procedimientos pueden definirse como: "un conjunto de acciones ordenadas, orientadas a la consecución de una meta". Ricardo (2007) plantea que esta meta o finalidad debe ser el resultado de un vínculo entre la necesaria capacitación de los educandos y las necesidades del entorno; se trataría de definir procedimientos que permitan al que aprende hacerlo "en", "desde" y "para" el medio ambiente.

La utilización de los procedimientos debe atender tanto a lo externo como a lo interno del proceso de enseñan- za-aprendizaje, de modo que, en correspondencia con la orientación, la ejecución y el control de la actividad de aprendizaje de los estudiantes se propicie la activación de los procesos psíquicos en las esferas cognitiva, afectiva y volitiva y que impliquen el análisis, la valoración, la generalización y que tengan en cuenta los momentos de la dirección de la actividad cognoscitiva, la motivación, la orientación, la ejecución y el control, así como los niveles de asimilación en correspondencia con las particularidades del contenido y el grupo docente.

De ahí que, entre los procedimientos metodológicos en el proceso de enseñanza-aprendizaje de la biología en secundaria básica se encuentran: 1) los lógicos, que atienden la actividad intelectual cognoscitiva y la creatividad de los alumnos; 2) los técnicos, asociados a aquellos métodos que requieren la utilización de medios de enseñanza; y 3) los organizativos, que permiten organizar la actividad cognoscitiva de los alumnos (Salcedo et al., 2002).

No obstante, para estimular las potencialidades de los educandos, en las dimensiones cognoscitiva, motivacional, metacognitiva, afectiva y axiológica se necesita incorporar al proceso docente-educativo de la biología un nuevo procedimiento que favorezca a la educación para la conservación de la biodiversidad. En este sentido, el aprendizaje cobra sentido en la medida en que, al construir su propio conocimiento, el aprendiz realiza procesos mentales que le permiten estar consciente de lo que aprende, cómo lo aprende y el fin para qué lo aprende, a la vez que se compromete con la autorregulación de su actividad de aprendizaje (Torrano et al., 2017).

En concordancia y de forma más específica en la presente investigación, se define el procedimiento didáctico de interconexión con el entorno ambiental como:

la vía para estimular el acercamiento y el reconocimiento de la biodiversidad del entorno ambiental, considerando los saberes entre la ciencia y el saber local para lograr la educación en la conservación, uso y manejo sostenible, así como el desarrollo de conocimientos, convicciones, valores, habilidades y la formación de actitudes que les permitan a los sujetos implicarse, de forma activa, protagonista y transformadora en el medio ambiente. (García, 2013, p. 73)

El procedimiento está estructurado en cuatro fases: observación, explicación, interpretación y comprensión. Cada una de ellas está representada por acciones invariantes que no constituyen un esquema rígido que los docentes deben seguir a la hora de planificar actividades de educación ambiental fuera del aula, ya que aunque son la guía 
general de acciones que deben realizar los estudiantes, se necesita el acompañamiento del docente como tutor durante la implementación de las sesiones de trabajo, para que las adapte a las características psicológicas y sociales de cada grupo de estudiantes, atendiendo a las tradiciones culturales de la comunidad donde viven y se desenvuelven, sus rasgos espirituales y afectivos, así como sus modos de vida, creencias, mitos, ideas, reglas, normas y sistemas de valores.

En la figura 1 se muestra el procedimiento diseñado y las fases que lo dinamizan.

Figura 1. Representación metodológica del procedimiento.

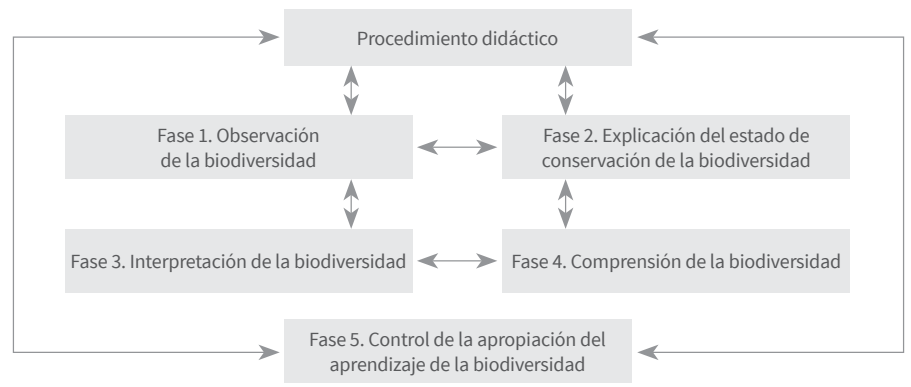

Fuente: elaboración propia.

El objetivo general del procedimiento propuesto es familiarizar al estudiante con la biodiversidad de su entorno ambiental próximo, con el cual interactúa y desde donde recibe las múltiples influencias que contribuyen a la adquisición de conocimientos y valores, así como al desarrollo de hábitos, habilidades, capacidades y actitudes en estrecho vínculo con la realidad ambiental donde vive y desarrolla.

Para la puesta en práctica del procedimiento, el docente debe ser consecuente con las formas organizativas de la enseñanza de la biología, fundamentalmente la clase de biología y la excursión docente biológica en su variante de excursión a la naturaleza o práctica de campo. Esta última forma de organización del proceso de enseñanza-aprendizaje de la biología posee un gran valor pedagógico, pues permite que el estudiante vincule la escuela con la vida, la teoría con la práctica y que asimile los conocimientos mediante la observación de los objetos y fenómenos en su propia realidad ambiental próxima. De igual manera, los prepara para la orientación en el terreno y para observar, diferenciar, establecer relaciones entre los fenómenos que acontecen en la naturaleza, localizar los organismos, desarrollar habilidades para el trabajo práctico y hábitos elementales de investigación en la naturaleza y contribuir a la formación de la concepción científica del mundo. Así, renuevan los modos para el acercamiento, el reconocimiento y la conceptualización de la biodiversidad (Trujillo y Valbuena, 2015).

En la figura 2 se presenta una metodología que detallada las etapas expuestas por Bosque (2002) para la realización de excursiones a la naturaleza.

Figura 2. Metodología para la excursión a la naturaleza.

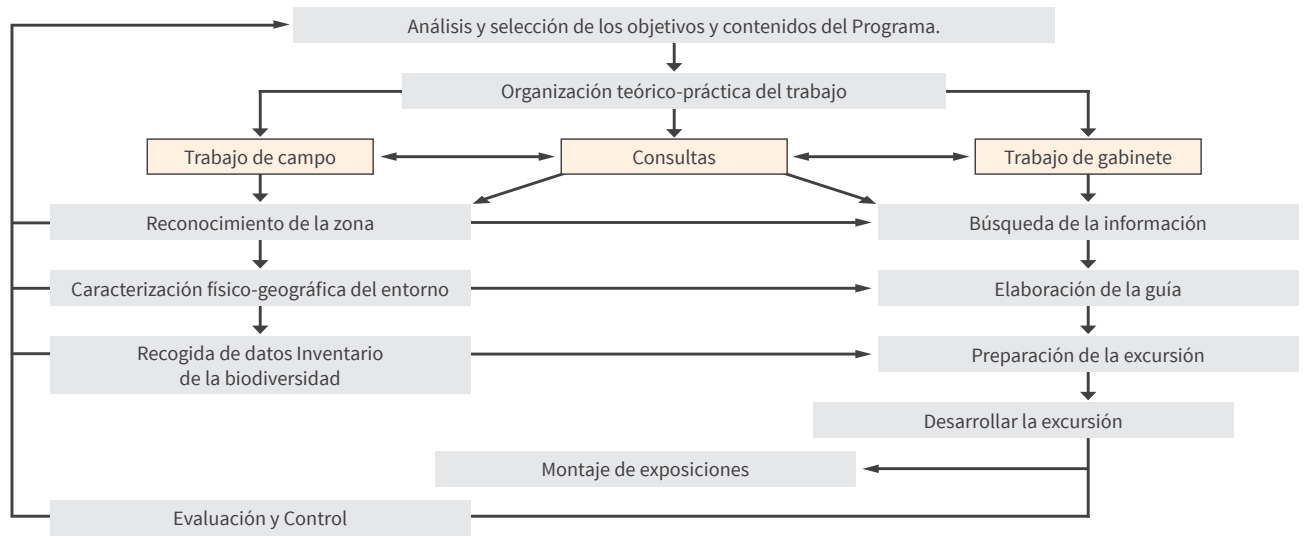

Fuente: Bosque (2002). 
A esta metodología agregamos los horarios de salida y de regreso, los puntos de interés didácticos dentro del itinerario o marcha-ruta, la selección y organización de los objetivos del grado y asignatura por tratar de la o las unidades didácticas del programa de Biología, los métodos de enseñanza, el procedimiento didáctico diseñado y las actividades que realizarán los estudiantes.

Esto presupone una correcta planificación de las medidas organizativas y de seguridad de los participantes, el tiempo de duración de la actividad, el tipo de vestuario, la organización de los equipos y los materiales por emplear (lápices, libretas, cámaras fotográficas, mapas, teléfono móvil, cuchillas, lupas, bolsas de nailon, recipientes, etc.)
En el desarrollo de la excursión, el docente de biología no se asume como el actor principal que aporta de manera unilateral información al recorrido, sino que es un actor que aprende y enseña la biodiversidad de la realidad ambiental junto con estudiantes participantes.

Una vez explicada la intención investigativa y las posibilidades que brinda la metodología para la excursión, en la tabla 1 se describen las fases del procedimiento diseñado y sus acciones invariantes, donde el estudiante al implicarse de manera activa y protagónica contribuye al mejoramiento de su propio aprendizaje.

Tabla 1. Procedimiento didáctico y sus acciones invariantes

\begin{tabular}{|c|c|}
\hline $\begin{array}{c}\text { Fases del } \\
\text { procedimiento }\end{array}$ & Acciones invariantes \\
\hline $\begin{array}{l}\text { Observación de la } \\
\text { biodiversidad }\end{array}$ & $\begin{array}{l}\text { - Identificar el objeto de observación y sus particularidades. } \\
\text { - Localizar en el mapa el área desde el punto de vista físico y geográfico. } \\
\text { - } \text { observar los principales tipos de paisajes; suelo; clima; temperatura; insolación; humedad relativa; } \\
\text { actividad diaria; vegetación; sustrato; estrato; refugios; sitios de descanso; reproducción y distribución de } \\
\text { las especies en los ecosistemas marinos y costeros. } \\
\text { - Describir oral y gráficamente las características del objeto de observación y distinguir los rasgos esenciales } \\
\text { que lo tipifican. }\end{array}$ \\
\hline $\begin{array}{l}\text { Explicación del estado } \\
\text { de conservación de la } \\
\text { biodiversidad }\end{array}$ & $\begin{array}{l}\text { - Reflexionar acerca de las relaciones causales y valorar la situación que posee el área seleccionada } \\
\text { (afectada, medianamente afectada y fuertemente afectada). } \\
\text { - Determinar los criterios sobre las causas y efectos de los cambios en la biodiversidad y la clasificación del } \\
\text { tipo de problema ambiental que se presenta el área de estudio. } \\
\text { - Ordenar los juicios de partida y razonamientos al resto del grupo para despertar la motivación y la } \\
\text { necesidad de la conservación, uso y manejo sostenible de la biodiversidad. } \\
\text { - Relacionar las interacciones entre los organismos y entre los ecosistemas del área de estudio ordenando } \\
\text { lógicamente las interrelaciones encontradas. }\end{array}$ \\
\hline $\begin{array}{l}\text { Interpretación de la } \\
\text { biodiversidad }\end{array}$ & $\begin{array}{l}\text { - Identificar conceptos básicos, como biodiversidad y conservación. } \\
\text { - Interacción directa docente-estudiantes para explorar sus potencialidades para enfrentarse a los diferentes } \\
\text { - Relacionar la representatividad de la biodiversidad. } \\
\text { - Sacar conclusiones acerca de los elementos, relaciones y razonamientos que aparecen en el objeto o } \\
\text { información por interpretar. } \\
\text { - Comunicar de forma oral y escrita sus juicios conclusivos. }\end{array}$ \\
\hline $\begin{array}{l}\text { Comprensión de la } \\
\text { biodiversidad }\end{array}$ & $\begin{array}{l}\text { - Comprender la importancia de la cultura popular (costumbres, religiosidad y tradiciones, mitos, historias } \\
\text { - } \text { Ie vida, leyendas). } \\
\text { - Compificar los principales problemas ambientales y sus causas. } \\
\text { - Valorar la representatividad de la diversidad biológica existente y sus principales amenazas. } \\
\text { - Relacionar los valores naturales, histórico-culturales y socioeconómicos de las áreas de estudio. } \\
\text { - Valorar las causas y consecuencias de la pérdida de la biodiversidad. } \\
\text { - Comunicar de forma oral y escrita sus juicios conclusivos. }\end{array}$ \\
\hline
\end{tabular}




\begin{tabular}{|c|c|}
\hline $\begin{array}{c}\text { Fases del } \\
\text { procedimiento }\end{array}$ & Acciones invariantes \\
\hline $\begin{array}{l}\text { Control de la apropiación } \\
\text { del aprendizaje de la } \\
\text { biodiversidad }\end{array}$ & $\begin{array}{l}\text { - Determinación de los objetos de evaluación (¿qué evaluar?) } \\
\text { conservación, así como el desarrollo de las habilidades para identificar, caracterizar y resolver problemas } \\
\text { sobre las afectaciones a la biodiversidad. } \\
\text { - Comprobar las valoraciones para promover acciones que garanticen la protección y conservación de la } \\
\text { biodiversidad. } \\
\text { - Establecer indicadores para medir el aprendizaje en la solución del problema, como la motivación, el } \\
\text { tiempo, la independencia y la creatividad. } \\
\text { - Evaluar las transformaciones en los modos de actuación. } \\
\text { - Proyectar la retroalimentación a partir de las dificultades. } \\
\text { - Elaborar preguntas escritas y orales que estimulen la búsqueda de la solución (para evaluar el lenguaje } \\
\text { escrito y oral) y determinar las tareas docentes que tiene que resolver (lenguaje escrito). } \\
\text { - Elaborar y discutir informes y/o ponencias (lenguaje escrito). } \\
\text { - Preparar la defensa de informes y/o ponencias por medio de dibujos y/o textos (lenguaje escrito y oral). } \\
\text { - Presentar y divulgar en los medios de comunicación, obras artísticas dibujos y/o textos relacionadas con la } \\
\text { biodiversidad del territorio. } \\
\text { - Evaluar el impacto de las acciones de divulgación y conservación. }\end{array}$ \\
\hline
\end{tabular}

Fuente: elaboración propia.

\section{Resultados y discusión}

A continuación, se presentan los aspectos generales encontrados a partir del análisis de las fases del procedimiento. Con la ayuda de los miembros del grupo se reconstruyó la experiencia vivida, su proceso interno, el contexto, para luego proyectarse y aportar a la descripción de diversos elementos que confluyen en la enseñanza aprendizaje de la biodiversidad. La aplicación de esta propuesta permitió examinar comparativamente la complejidad estructural de los argumentos formulados antes y después de la intervención didáctica.

De esta manera, los estudiantes, valiéndose del trabajo cooperativo, son los responsables de redactar un informe al final de la actividad y, por tanto, de fijar los objetivos de su propio aprendizaje (Santos-Ellakuria, 2019). El profesor, en función del escenario provisto, establece el cronograma de trabajo y la fecha de entrega (que será al finalizar la excursión), y funge como guía en el aprendizaje personal, pero la carga de solucionar el problema recae exclusivamente en los estudiantes. El docente sugiere a los participantes recurrir a las experiencias vividas durante el recorrido para redactar la evaluación. La extensión del trabajo será de no más de tres páginas (incluidas las fotografías) y deberá entregarse en formato impreso.

Presentados los elementos anteriores, se muestran los resultados puntuales por fases.

\section{Primera fase: Observación de la biodiversidad}

En esta fase, los estudiantes implicados desarrollaron las habilidades de observación de los diversos ecosistemas del área seleccionada, incluyendo los marinos, como los arrecifes coralinos y los pastos marinos (estos últimos conocidos en Cuba como seibadales), los fondos arenosos (que incluyen las playas), los fondos fangosos y los fondos rocosos de macrolaguna y las lagunas costeras. Especial significación para los participantes tuvo el ecosistema de manglar, por su abundancia en biodiversidad y el papel que desempeña en la protección de la zona costera.

Se observaron, además, los tipos de plantas y aves representativas del área de estudio clasificándolas a partir del tipo de alimentación: granívora, insectívora, carnívora y frugívora, así como el tipo de suelo, vegetación donde habitan y sus principales amenazas. En consecuencia, durante el recorrido los participantes anotaron lo observado y definieron la representatividad de la biodiversidad del área como autóctona, endémica, introducida, exótica invasora, migratoria, cultivada, etc.; al igual que la categoría de conservación.

\section{Segunda fase: Explicación del estado de conservación de la biodiversidad}

Como resultados del diagnóstico aplicado a los estudiantes implicados en la muestra se constató que estos explicaban la importancia de las plantas y animales solo desde su significación para la naturaleza, la medicina, la alimentación, la economía y la industria. 
A partir de la utilización del procedimiento se revierte esta limitación al explicar el vínculo de la biodiversidad con otros servicios ecosistémicos que ella ofrece, como los servicios culturales (valores espirituales y religiosos, educativos, estéticos, recreativos, simbólicos, cognitivos, históricos y en la vida afectiva del ser humano, entre otros).

\section{Tercera fase: Interpretación de la biodiversidad}

Al abordar la definición de la biodiversidad los estudiantes solo hacían referencias a la diversidad de animales y plantas existentes en los ecosistemas. Una vez empleado el procedimiento en la práctica se aprecia cómo interpretan el significado de la biodiversidad y que esta rebasa los planteamientos manifestados inicialmente; reconocen que representa la vida en todas sus manifestaciones, expresada en genes, especies (incluyendo la humana y su diversidad cultural).

Se logran avances en la identificación de las amenazas de la biodiversidad en el área seleccionada y la representatividad de las especies autóctonas y endémicas, se realizan actividades de educación ambiental, como la recogida de plásticos en la zona costera y charlas educativas ambientales.

\section{Cuarta fase: Comprensión de la biodiversidad}

Los estudiantes logran comprender la integridad de los problemas ambientales a nivel global, nacional y local, así como la naturaleza compleja de la biodiversidad, resultante de la interacción de sus aspectos biológicos, físicos, históricos, sociales y culturales. A esto se suma la comprensión de las potencialidades de la diversidad biológica del contexto local, sus realidades, su riqueza natural y su estado de conservación, lo que se traduce en la valoración de su contexto, no solo por lo que brinda, sino por lo que es y los significados que encierra para su tradición, cultura, rasgos espirituales, creencias, mitos, ideas, reglas, normas y sistemas de valores, el reconocimiento de lo patrimonial y su entramado social. Esto permite establecer un diálogo de saberes entre la ciencia y el saber local.

\section{Quinta fase: Control de la apropiación del aprendizaje de la biodiversidad}

En la evaluación del aprendizaje se logró que esta tuviera un carácter procesal y que combinara lo cualitativo y lo cuantitativo y se practicara mediante la coevaluación, la heteroevaluación y la autoevaluación metacognitiva, para lograr que el estudiante asuma la responsabilidad sobre su propio aprendizaje, reflexionando acerca de su implicación en el trabajo grupal y los resultados obtenidos durante las salidas a la naturaleza y con ello, establecer la relación entre las ideas previas y los nuevos conceptos biológicos, como los de biodiversidad y educación para la conservación.

Se evidenció el desarrollo de un saber conceptual, que ha progresado, ha sido pertinente y ha tenido un significado personal para el estudiante.

Se logró además, estimular la curiosidad, la motivación y el mayor acercamiento del estudiante a la biodiversidad de la realidad ambiental próxima a través de las excursiones a la naturaleza, propiciar la independencia cognoscitiva y el rol protagónico del estudiante en el proceso de estudio a través de una experiencia de aprendizaje fuera del aula, potenciar el grado de concienciación respecto al perjuicio socioambiental que supone la pérdida de la biodiversidad y contribuir al conocimiento de la biodiversidad autóctona y endémica del territorio y la transformación de los modos de actuación y respeto a las diferentes formas de vida.

De esta manera, los resultados de la experiencia vivida por los estudiantes implicados fueron divulgados en los medios de comunicación, matutinos, concursos de conocimientos de la escuela, jornadas científicas, obras de teatro, entre otras iniciativas.

\section{Conclusiones}

La concepción del procedimiento didáctico está dirigida, entre otros aspectos, a desarrollar y favorecer una actitud de curiosidad e investigación, propiciar la comprensión de la biodiversidad y de su lugar en la naturaleza, así como de la unidad y la diversidad de los seres vivos, promover respeto y sentimiento hacia todas las formas de vida que habitan en el entorno ambiental, enseñar el arte de participar en investigaciones científicas, formular preguntas, diseñar experimentos y desarrollar el método investigativo de la enseñanza problémica en la actividad práctica.

La propuesta constituye una alternativa viable que garantiza perfeccionar la preparación metodológica de los profesores de biología de la escuela media para elevar la calidad de la dirección del proceso de enseñanza-aprendizaje de la biología en función de lograr la actualización científica del estudiante y un adecuado tratamiento del contenido de biodiversidad desde un enfoque holístico, 
interdisciplinar, ecológico, integrador, sistémico, bioético, ecosistémico, evolutivo, ético y socioeconómico, que favorezca en el estudiante la educación para la conservación y el uso sostenible de la biodiversidad.

De esta manera, el procedimiento resulta pertinente y efectivo para mejorar el aprendizaje de la biodiversidad fuera del aula y se validan satisfactoriamente por la muestra a partir de los instrumentos utilizados (mediante la observación, encuestas y entrevistas). Se logran, además, altos niveles de motivación y transformación de los conocimientos, los sentimientos, las actitudes y las convicciones con relación a la biodiversidad del medio natural próximo y la problemática en torno a la educación para la conservación, así como el fortalecimiento de los valores identitarios del patrimonio histórico-natural local con el que interactúan. Esto corrobora la factibilidad de la propuesta.

La excursión a la naturaleza como una forma de organización del proceso de enseñanza-aprendizaje de la biología, que se realiza fuera del aula con objetivos docentes bien definidos, se concibe como un ejercicio de reflexión en constante construcción, y el espacio educativo y pedagógico que es capaz de transformar y redescubrir realidades cercanas y establecer alianzas entre los estudiantes y los espacios naturales o creados artificialmente por el hombre, para potenciar la formación de valores y la adquisición de conocimientos de los procesos geográficos, fenómenos, hechos y objetos de su realidad ambiental próxima. Mediante ella se establece la interacción constante con los seres vivos; además, se crean las condiciones favorables para la formación en el estudiante de la cultura ambiental, elevar las cualidades de la personalidad como el trabajo en equipo, la solidaridad, la organización de la actividad práctica y la responsabilidad ante el medio ambiente.

\section{Referencias}

Álvarez, A. (2001). De la herencia cotidiana al tesoro perdido: nuevos desafíos de la educación ambiental para la conservación de la biodiversidad. Interciencia Venezuela, 26(10), 429-433. http:// www.scielo.org.ve/

Bosque, R. (2002). La excursión docente en la Educación Primaria: una propuesta para el perfeccionamiento de su realización. [Tesis de doctorado, Universidad de Ciencias Pedagógicas].
Castro, A. y Valbuena, É. (2018). Algunas relaciones entre la autonomía de la biología y la emergencia de su didáctica: consideraciones sobre la complejidad de enseñar una ciencia compleja. Ciênc. Educ. Bauru 24(2), 267-282. https://doi. org/10.1590/1516-731320180020002

Colmenares, A. (2011). Investigación-acción participativa: una metodología integradora del conocimiento y la acción. Revista Latinoamericana de Educación, 3(1), 102-115. https://revistas. uniandes.edu.co/doi/pdf/10.18175/vys3.1.2012.07

García, O. (2013). Metodología orientada al tratamiento del contenido de biodiversidad en la enseñanza de la biología en Secundaria Básica. [Tesis de doctorado, Universidad de Ciencias Pedagógicas].

Gil Álvarez, J. L., León González, J. L. y Morales Cruz, M. (2017). Los paradigmas de investigación educativa, desde una perspectiva crítica. Conrado, 13(58), 72-74. https://conrado.ucf.edu. cu/index.php/conrado/article/view/476

Herrera, M. (2020). Saberes acerca de la biodiversidad en un escenario de educación no convencional. Bio-grafía, 11(22), 121-132. https://revistas. pedagogica.edu.co/index.php/bio-grafia/article/ view/11593

Labarrere, G. y Valdivia, G. (1989). Pedagogía. Pueblo y Educación.

Novo, M. (1998). La educación ambiental: bases éticas, conceptuales y metodológicas. Universitas.

Programa de las Naciones Unidas para el Medio Ambiente-Pnuma. (2005). Manual de ciudadanía ambiental global: Diversidad biológica.

Ricardo, D. (2007). Procedimiento metodológico para desarrollar la sensibilidad estética ambiental en la Secundaria Básica. [Tesis de doctorado inédita, Instituto Superior Pedagógico José Martí].

Salcedo, I., Hernández, J., del Llano, M., McPherson, M. y Daudinot, I. (2002). Didáctica de la biología (2. ${ }^{a}$ ed.). Pueblo y Educación.

Santos-Ellakuria, I. (2019). Propuesta para mejorar la didáctica de la biodiversidad en la asignatura de Biología y Geología de $4 .^{\circ}$ de Eso. Ikastorratza. e-Revista de Didáctica, 22, 90-121. http://www.ehu. es/ikastorratza/22_alea/6.pdf 
Silvestre, M. (2003). Aprendizaje, educación y desarrollo. Pueblo y Educación.

Torrano, F.; Fuentes, J. L. y Soria, M. (2017). Aprendizaje autorregulado: estado de la cuestión y retos psicopedagógicos. Perfiles Educativos, 39(156), 160-173. http://www. scielo.org.mx/scielo.php?script=sci_arttext\&pid=S0185-26982017000200160

Trujillo, L. y Valbuena, É. (2015). ¿Educación no convencional, no formal o informal? Emergencia en el marco de investigación con tres licenciados en biología. Biografía (edición extraordinaria), 1-11. 